\section{A metabolomics-based biomarker signature discriminates pancreatic cancer from chronic pancreatitis}

\author{
Eithne Costello
}

The incidence of pancreatic ductal adenocarcinoma (PDAC) is increasing. The projection that it will surpass breast cancer to become the second-leading cause of cancer-related deaths by 2030 serves as a wake-up call to stakeholders, including healthcare systems and researchers. ${ }^{1}$ Earlier diagnosis is one factor that could alter this trajectory. Correspondingly, the research community has made substantial efforts to find biomarkers that will enable the diagnosis of pancreatic cancer at a stage where it can be successfully treated. ${ }^{2}$ There are however, significant challenges. Pancreatic cancer is a very heterogeneous disease with great interindividual variation, as well as significant heterogeneity within the tumours of individuals. ${ }^{34}$ This calls for large sample sizes to ensure adequate representation of subtypes. Moreover, biomarker development programmes require samples to be separated into independent training and test sets, further increasing the quantity of samples needed. However, the number of new cases of pancreatic cancer per year is low compared with other common cancers. Therefore, in order to acquire

Correspondence to Professor Eithne Costello, Department of Molecular and Clinical Cancer Medicine, Institute of Translational Medicine, University of Liverpool, 2nd Floor Sherrington Building, Ashton Street, Liverpool L69 3GE, UK; ecostell@ liverpool.ac.uk sufficient samples of patients with cancer and control individuals for biomarker studies, multicentre collaborations are essential. Such collaborations require orchestration, as the use of standardised protocols for collection and storage of both case and control samples across centres minimises bias. Indeed, the performance of biomarkers may be affected by a significant number of variables, such as age, gender, sample collection method, duration of sample storage and sample handling.

Additional sources of bias related to pancreatic cancer biomarker development have emerged in recent years. For example, the presence of obstructive jaundice, whether the result of cancer or from benign causes is associated with higher levels of certain blood proteins. ${ }^{5-7}$ The significance of this finding is that obstructive jaundice may place the validity of biomarker data at risk. Essentially, it was observed that the presence of obstructive jaundice in patients with PDAC increased the sensitivity of candidate biomarkers for the detection of PDAC over controls and thereby contributed false-positive findings. ${ }^{5}$ Understanding comorbidities and how they affect the performance of biomarkers is also important. Some $45 \%$ to $65 \%$ of patients with PDAC have PDAC-associated diabetes mellitus. ${ }^{8}$ This condition may contribute to the heterogeneity in performance of candidate biomarkers. ${ }^{9}$ If diabetes mellitus is not taken into consideration, then potentially valuable markers may be dismissed, even though they may facilitate the detection of a subset of patients with PDAC, such as those with diabetes or those without diabetes.

Undoubtedly, one of the greatest biomarker-related challenges in this field is finding biomarkers that accurately distinguish PDAC from other diseases of the pancreas, where overlapping signs and symptoms make differential clinical diagnosis difficult. Indeed, many promising candidate biomarkers, which are excellent at differentiating healthy individuals from patients with PDAC, show disappointing specificity when challenged to discriminate patients with PDAC from patients with chronic pancreatitis (CP). Although some small-scale studies have shown promise, the general performance of biomarkers including that of the gold standard cancer antigen 19-9 (CA19-9) in differentiating PDAC from CP is modest, and improvements are needed. In this issue, Mayerle, Lerch and colleagues describe a breakthrough in this respect, with the identification of a metabolite-based biomarker signature which distinguishes PDAC from CP with much greater accuracy than is achieved by CA19-9 alone.

Metabolomics, the study of metabolites or 'chemical fingerprints' related to specific cellular processes, is emerging as a formidable means of detecting disease-induced alterations in metabolism. In this issue, Mayerle and coworkers ${ }^{10}$ describe global analysis of blood metabolites including lipids to find candidate metabolites that distinguish PDAC from CP. The study was 
designed to accurately exclude suspected pancreatic cancer in patients with CP, with emphasis placed on optimising the negative predictive value (NPV).

An impressive 914 subjects were recruited from three German centres and samples were divided into exploratory, training and test sets. Gas chromatography-mass spectrometry and liquid chromatographytandem mass spectrometry were undertaken to generate metabolomic profiles of serum or plasma. This led to the identification of 477 metabolites which fell into 10 ontology classes, the majority belonging to lipids, fatty acids and related metabolites. A total of 29 metabolites were significantly altered between PDAC and CP in serum and plasma of the training set. Subsequently, an Elastic Net algorithm, applied to plasma samples from the training set of 158 subjects $(\mathrm{n}=78$ PDAC; $\mathrm{n}=80 \mathrm{CP})$ identified nine metabolite signature plus CA19-9 which, in the training set, achieved an impressive area under the receiver operating characteristic curve of 0.96 in discriminating PDAC from CP. The NPV was $99.9 \%$, assuming a cumulative incidence of $1.95 \%$ of PDAC in the CP population. The performance of the biomarker signature was similarly effective in an independent test set of plasma samples with a resultant NPV of 99.8\%. Reassuringly, when the biomarker panel was challenged to discriminate resectable pancreatic cancer from CP, it did so with high accuracy in both training and test sets. Thus, the biomarker signature is effective at a time point in the disease pathway when surgical intervention is an effective treatment option.

Given that the signature was derived from training on PDAC and CP samples, it was interesting to know whether it could distinguish patients with PDAC from control subjects devoid of pancreatic disease. It emerged that the distinguishing features of the biomarker signature related to PDAC, rather than $\mathrm{CP}$, as biomarker scores for CP overlapped with the non-pancreatic disease controls. In test sets, the biomarker signature was not superior to CA19-9 at distinguishing either PDAC or resectable PDAC from non-pancreatic controls. Thus, consistent with the aim of the study, the strength of the biomarker signature lies in its ability to distinguish PDAC from CP and in the high NPV achieved. Notably, the biomarker signature incurred fewer false positives and detected more true positives than CA19-9 alone.

In summary, the authors recruited patients from three centres in Germany to achieve a uniquely large study, in which extensive metabolite analysis led to a trained and validated biomarker signature that accurately discriminates PDAC from CP. This study uncovers the potential of metabolomics for the diagnosis of PDAC and indeed for cancer diagnostics in general.

Competing interests None declared.

Provenance and peer review Commissioned; internally peer reviewed.

(c) Article author(s) (or their employer(s) unless otherwise stated in the text of the article) 2018. All rights reserved. No commercial use is permitted unless otherwise expressly granted.

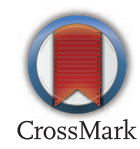

To cite Costello E. Gut 2018;67:2-3.

Received 8 February 2017
Revised 10 April 2017

Accepted 11 April 2017

Published Online First 24 May 2017

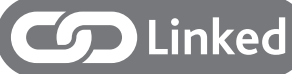

http://dx.doi.org/10.1136/gutjnl-2016-312432

Gut 2018;67:2-3.

doi:10.1136/gutjnl-2016-313665

\section{REFERENCES}

1 Rahib L, Smith BD, Aizenberg R, et al. Projecting cancer incidence and deaths to 2030: the unexpected burden of thyroid, liver, and pancreas cancers in the United States. Cancer Res 2014;74:2913-21.

2 Jenkinson C, Earl J, Ghaneh P, et al. Biomarkers for early diagnosis of pancreatic cancer. Expert Rev Gastroenterol Hepatol 2015;9:305-15.

3 Campbell PJ, Yachida S, Mudie LJ, et al. The patterns and dynamics of genomic instability in metastatic pancreatic cancer. Nature 2010;467:1109-13.

4 Yachida S, Jones S, Bozic I, et al. Distant metastasis occurs late during the genetic evolution of pancreatic cancer. Nature 2010;467:1114-7.

5 Tonack S, Jenkinson C, Cox T, et al. iTRAQ reveals candidate pancreatic cancer serum biomarkers: influence of obstructive jaundice on their performance. Br J Cancer 2013;108:1846-53.

6 Yan L, Tonack S, Smith R, et al. Confounding effect of obstructive jaundice in the interpretation of proteomic plasma profiling data for pancreatic cancer. J Proteome Res 2009;8:142-8.

7 Nie S, Lo A, Wu J, et al. Glycoprotein biomarker panel for pancreatic cancer discovered by quantitative proteomics analysis. J Proteome Res 2014;13:1873-84

8 Pannala R, Leirness JB, Bamlet WR, et al. Prevalence and clinical profile of pancreatic cancerassociated diabetes mellitus. Gastroenterology 2008;134:981-7.

9 Jenkinson C, Elliott VL, Evans A, et al. Decreased serum thrombospondin-1 levels in pancreatic cancer patients up to 24 months prior to clinical diagnosis: association with diabetes mellitus. Clin Cancer Res 2016;22:1734-43.

10 Mayerle J, Kalthoff H, Reszka R, et al. Metabolic biomarker signature to differentiate pancreatic ductal adenocarcinoma from chronic pancreatitis. Gut 2018;67:128-37. 